\title{
COMMISSIONING STATUS OF THE KEKB LINAC
}

\author{
Y.Ogawa*, Linac Commissioning Group (LCG) ${ }^{\#}$ \\ KEK, 1-1 Oho, Tsukuba, Ibaraki 305-0801 Japan
}

\begin{abstract}
After entering into the full-commissioning phase of the KEKB linac in May, 1998, we have established two linac injection modes for the KEKB ring: an 8-GeV electron beam and a $3.5-\mathrm{GeV}$ positron beam, as well as beam modes for the rings of the synchrotron light sources. All modes are easily switched for almost one minute without any trouble. The linac beam characteristics have been substantially improved by introducing several fine-tuning procedures, while key features concerning stable operation have been pursued by employing, for instance, energy-feedback loops. We report on the present status of the linac commissioning while especially emphasising the operational characteristics.
\end{abstract}

\section{INTRODUCTION}

The KEK electron-positron linac is the injector for two asymmetric rings (8-GeV electron and $3.5-\mathrm{GeV}$ positron rings) of the KEK B-factory (the KEKB linac is named just after this), as well as for two different rings (2.5-GeV electron rings) of the Photon Factory (PF). It has been pointed out that since beam-mode switching among various kinds of beams should be quite frequent, perfect reproducibility of each beam could be one of the key issues for the successful operation of the KEKB ring [1], which requires frequent and speedy injection for highluminosity collisions.

Full-commissioning of the KEKB linac [2] was started in May, 1998, while continuing ordinary injection into the $\mathrm{PF}$ ring. The principal goal of the full commissioning was to accomplish stable operation for injection to the KEKB rings as well as to assure efficient injection to two $\mathrm{PF}$ rings. The beam reproducibility has been pursued mainly regarding three aspects: the establishment of reliable beam-mode switching, various improvements of the beam quality, and beam-stability issues. In the following, the present status of the linac commissioning is reported while placing particular emphasis on these issues.

\section{BEAM-MODE SWITCHING}

\subsection{Beam Modes}

In Table I, two beam modes for KEKB and two modes for $\mathrm{PF}$ are listed, showing a wide variety of beam characteristics: a beam energy of 2.5 to $8 \mathrm{GeV}$ and a beam charge of 0.3 to $10 \mathrm{nC}$. Beam-mode switching among these modes requires a change of at least several hundred parameters: the magnetic field strength for the beam optics and orbit, the accelerating rf phases and acceleration/standby modes of the klystrons for the energy and energy spread, the feedback parameters for beam stability, the beam-monitor settings for beam diagnosis and so on.

Table 1: Beam-mode specifications

\begin{tabular}{|l|l|l|}
\hline \multicolumn{1}{|c|}{ Mode } & Energy & \multicolumn{1}{c|}{ Charge } \\
\hline KEKB e & $8.0 \mathrm{GeV}$ & $1.2 \mathrm{nC}$, single bunch \\
\hline KEKB e$^{+}$ & $3.5 \mathrm{GeV}$ & $\begin{array}{l}10 \mathrm{nC} \text { for primary e } \\
0.64 \mathrm{nC} \text { for } \mathrm{e}^{+}, \text {single bunch }\end{array}$ \\
\hline PF1 & $2.5 \mathrm{GeV}$ & $0.3 \mathrm{nC}$, multibunch \\
\hline PF2 & $2.5 \mathrm{GeV}$ & $0.5 \mathrm{nC}$, multibunch \\
\hline
\end{tabular}

\subsection{Switching Beam Modes}

A beam-mode-switching panel (Fig. 1) has been prepared so that all of the parameters can be easily and quickly changed among the beam modes. For the present, we are using two electon guns for the KEKB and PF beams, respectively, which was inevitable because we had to continue injection to the PF rings even during construction of the KEKB linac [3]. This situation makes beam switching between the KEKB and PF beams quite complicated, though this situation will be improved in the future. Since the energy ranges from 2.5 to $8 \mathrm{GeV}$ and the field strength of quadrupole magnets must be correspondingly changed, we have introduced a simple standardization of the magnets so as to avoid any hysteresis effects. This procedure mainly determines an overall time for switching of almost 1 minute. A reliability check has been carried out not only by examining the beam characteristics along the linac, but also by actually injecting each beam into the corresponding ring. It has turned out that beam switching, itself, in a short period is quite reliable, although a longterm drift of the beam characteristics has been observed (see section 4).

\footnotetext{
*Email: yujiro.ogawa@kek.jp

${ }^{\text {"N }}$. Akasaka, A. Enomoto, J. Flanagan, H. Fukuma, Y. Funakoshi, K. Furukawa, T. Ieiri, N. Iida, T. Kamitani, T. Kawamoto, M. Kikuchi, H. Koiso, S. Michizono, T. T. Nakamura, Y. Ogawa, Y. Ohnishi, S. Ohsawa, K. Oide, K. Satoh, M. Suetake, T. Suwada
} 


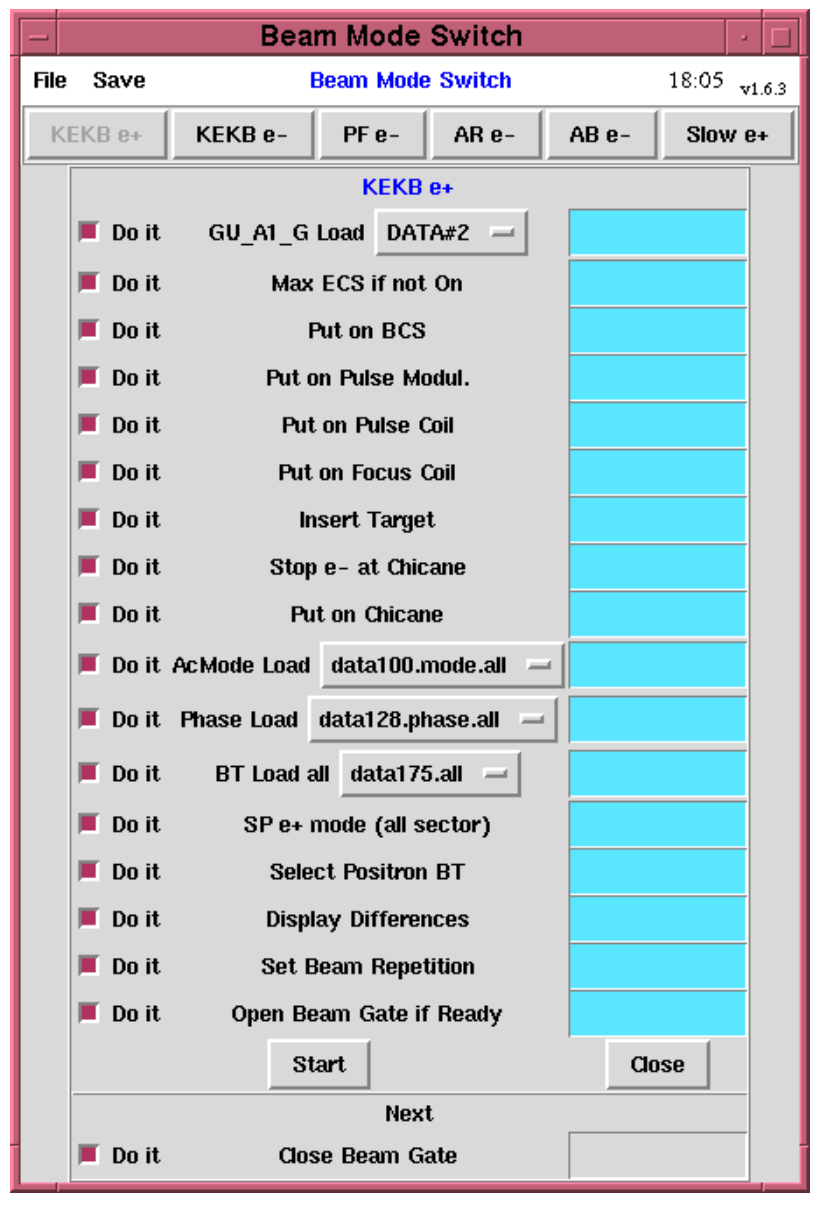

Figure 1: Beam-mode switching panel.

\section{IMPROVEMENTS OF BEAM QUALITY}

\subsection{Software Feedback Loop}

Some software feedback loops have been introduced in order to stabilise the gun energy, the powers of two subharmonic bunchers (SHB) and the beam energy and the orbits at the J-arc section $(1.5 \mathrm{GeV})$ and at the end of the linac, while hardware feedback loops are implemented for the rf phase-lock system in the SHBs. Fig. 2 shows an example of the feedback panels [4]. The time constant of the software feedback is over several seconds. As a result, the medium-period drift during beam injection was greatly improved. A sudden large variation of the quantities, however, which is discussed in the next section, can not be cured by this feedback, simply because the dynamic range is limited by various boundary conditions.

\subsection{Wire Scanners for Matching}

There are several regions where optics matching is necessary: at the exit of the bunching section, before/after the J-arc section and at the end of the linac. In order to facilitate and automate this matching, sets of a wire scanner system comprising at least three monitors at appropriate intervals in each section were installed and hardware-tested to measure the beam size [5]. Recently, the software, which nearly automates the optics matching as well as the beam-size measurement, has been completed and beam-tested at the beam-transport line to the KEKB ring. The results so far obtained are quite satisfactory and will be applied to the above-mentioned section at the linac.
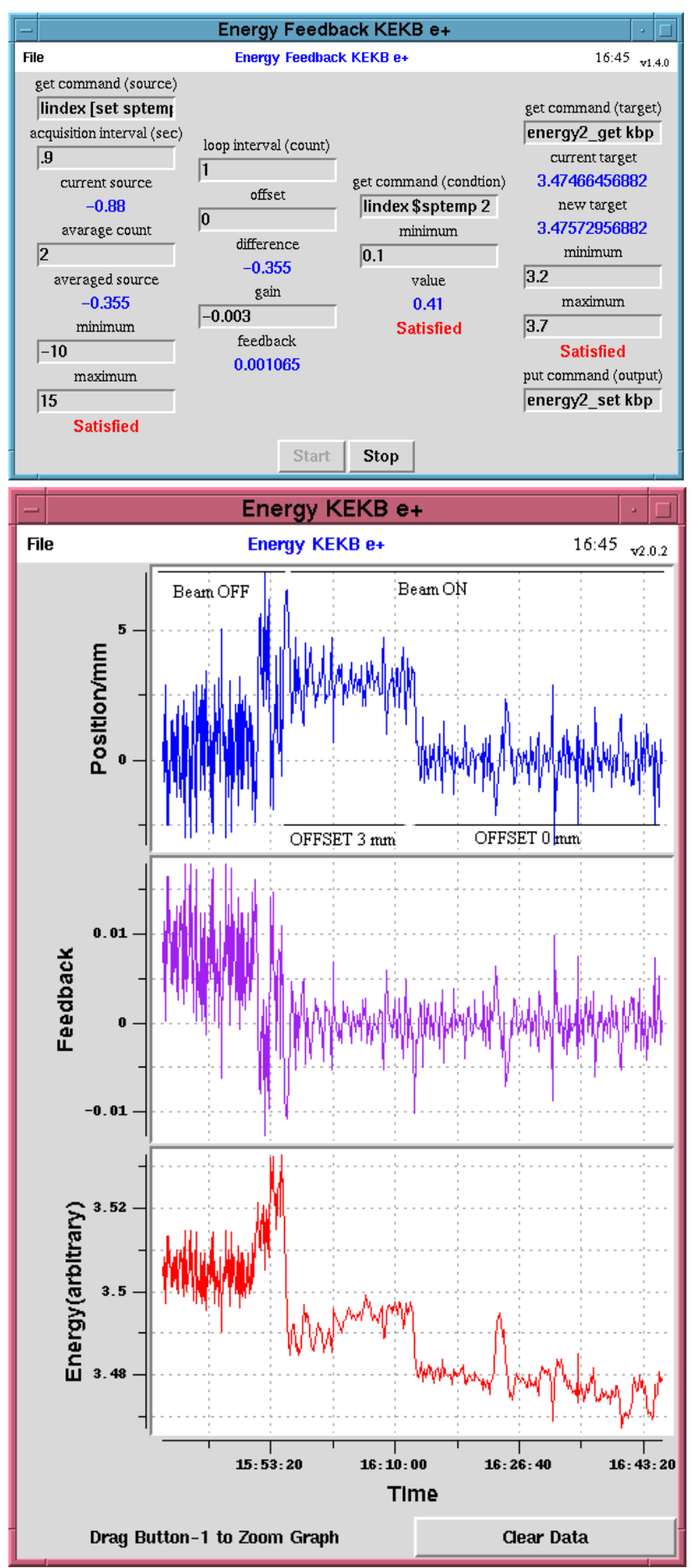

Figure 2: Example of the feedback panels. (upper: control panel, lower: status log) 


\section{STABILITY ISSUES}

The beam-stability issues in our linac are somehow complicated in that we must accomplish stable operation of different kinds of beams repeating a frequent beam switching. A simple classification concerning the stability, such as long- or short-term variations, is not clear, since only one beam can not be continuously observed for monitoring. It may happen that the status of some equipment changes, even during beam switching. In this case, for instance, it is not easy to identify the sources of variations by observing just the status of different beams. In this connection, various efforts to search for the sources of variation have been pursued through deriving information from the status of not only the beams, but also all equipment.

\subsection{Search for Sources of Orbit Variation by Singular Value Decomposition (SVD)}

The technique of singular value decomposition (SVD) [6] was applied to search for the sources of the orbit variation from the exit of the bunching section to the two upstream sectors of the linac. The matrix comprises time sets of the row vectors consisting of the signals of many beamposition monitors (BPM) along the linac. Figure 3 shows an example of the orbit patterns corresponding to the largest singular value. It seems that the sources of orbit variation originate just at the beginning of the linac. We have tried to identify them by checking the corresponding equipment, but have not yet succeeded.

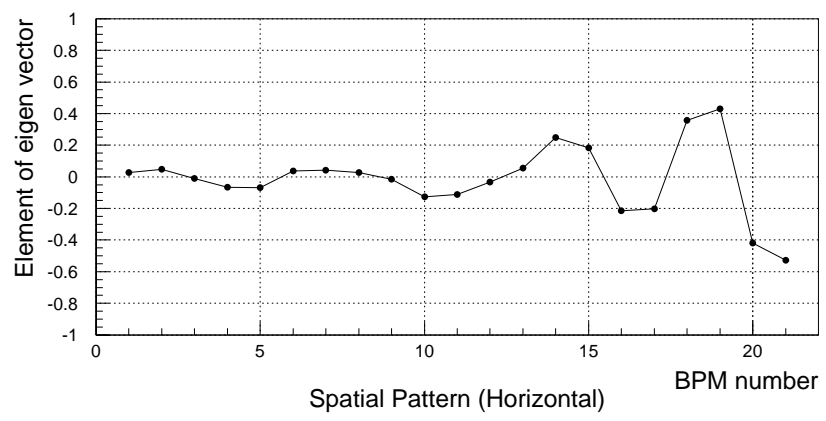

Figure 3: Example of the orbit patterns corresponding to the largest singular value by the SVD method.

\subsection{Reinforcement of Stability-Monitoring System}

In order to identify the sources of variation, a stabilitymonitoring system is being prepared. One of the most essential systems is an rf phase-monitoring system. For the moment, this system works only in three upstream sections, but has already been shown to be a quite powerful tool for stability monitoring. For instance, it has turned out that the phase of the first klystron for the bunching section sometimes varies by a few degrees (Fig. 4 ), possibly due to the environmental temperature.
Another example of a new monitoring system is a fast BPM detection system [7], in which data-taking at a beam repetition rate of $50 \mathrm{~Hz}$ can be realized. A FFT-analysis of the data taken at a point of large dispersion in the $\mathrm{J}$-arc section suggests the existence of energy variations, rather than simple orbit fluctuations, in the upstream sections. A statistical treatment, such as a correlation analysis, for all data taken by the above-mentioned system is being applied in order to figure out what is happening in the linac.

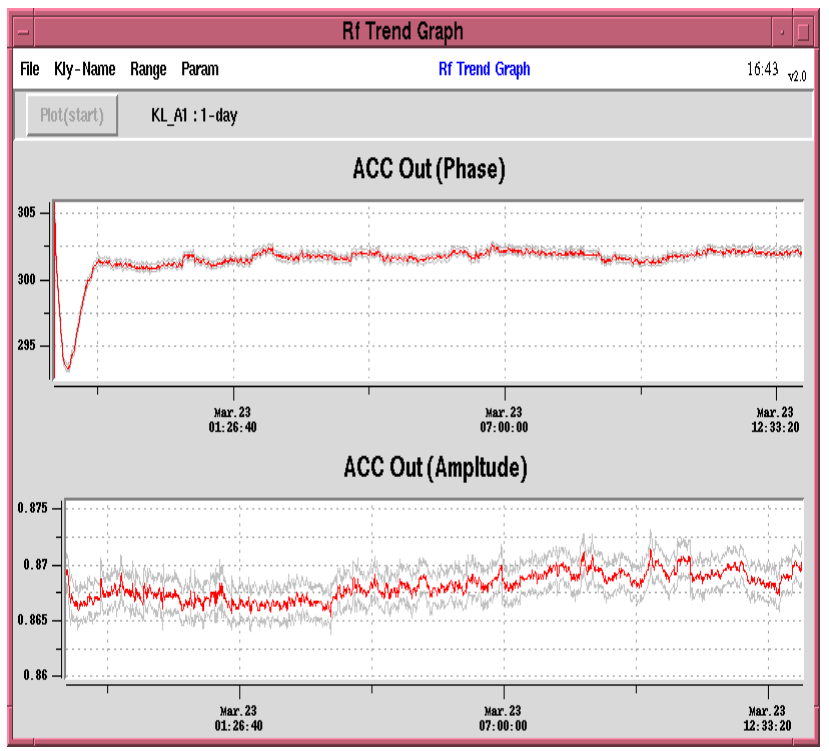

Figure 4: Observed phase variation of the first klystron for the bunching section (upper).

\section{CONCLUSIONS}

Two linac injection modes for the KEKB rings and those for the Photon Factory rings have been established. Beam-mode switching among four kinds of beams has been successfully verified by injecting a beam into each ring, employing various beam-tuning procedures, including feedback loops. The operation experience so far indicates that there are some stability issues to be figured out in order to maintain a high-quality beam. Many efforts are being made to identify the sources of variations.

\section{REFERENCES}

[1] K. Oide, in these proceedings.

[2] Y. Ogawa, "Commissioning of the KEKB Linac", to be published in Proc. of LINAC98, Chicago, August 23-28, 1998.

[3] A. Enomoto, "Commissioning of the KEKB 8-GeV e / 3.5-GeV e Injector Linac", Proc. of EPAC98, Stockholm, June 22-26, 1998.

[4] K. Furukawa et al., in these proceedings.

[5] Y. Funakoshi et al., in these proceedings.

[6] Y. Ohnishi et al., private communication.

[7] T. Suwada, private communication. 